\title{
Disponibilidade de metais pesados em Latossolo com aplicação superficial de escória, lama cal, lodos de esgoto e calcário
}

\author{
Juliano Corulli Corrêa ${ }^{(1)}$, Leonardo Theodoro Büll(1), Wanderley da Silva Paganini(2) e Iraê Amaral Guerrini(1)
}

(1)Universidade Estadual Paulista, Faculdade de Ciências Agronômicas, Fazenda Experimental Lageado, Caixa Postal 237, CEP 18603-970 Botucatu, SP. E-mail: correajc@superig.com.br, bull@fca.unesp.br, iguerrini@fca.unesp.br (2)Universidade de São Paulo, Faculdade de Saúde Pública, Avenida Dr. Arnaldo, no 715, Cerqueira César, CEP 01246-904 São Paulo, SP. E-mail: wsilvapa@sabesp.com.br

Resumo - O objetivo deste trabalho foi avaliar alguns atributos químicos do solo e a disponibilidade de cádmio $(\mathrm{Cd})$, cromo $(\mathrm{Cr})$, níquel $(\mathrm{Ni})$, mercúrio $(\mathrm{Hg})$, chumbo $(\mathrm{Pb})$ e arsênio $(\mathrm{As})$, por meio da extração pelo DTPA, em consequiência da aplicação superficial de escória de aciaria, lama cal e lodos de esgoto centrifugados e de biodigestores, nas doses 0 (testemunha), 2, 4 e $8 \mathrm{Mg} \mathrm{ha}^{-1}$ e um tratamento adicional composto pela calagem superficial na dose $2 \mathrm{Mg} \mathrm{ha}^{-1}$. O experimento foi conduzido em delineamento de blocos ao acaso, em condições de campo, em área sob sistema plantio direto, durante 2003 e 2004. A aplicação superficial de escória de aciaria, lama cal, lodo de esgoto centrifugado e de biodigestor, até a dose $8 \mathrm{Mg} \mathrm{ha}^{-1}$, assim como o calcário na dose $2 \mathrm{Mg} \mathrm{ha}^{-1}$, não trazem problemas de disponibilidade ao ambiente, com relação aos metais pesados $\mathrm{Cd}, \mathrm{Cr}, \mathrm{Hg}, \mathrm{Pb}$, $\mathrm{Ni}$ e As, quando aplicados sobre a superfície em Latossolo Vermelho distrófico, no sistema plantio direto. A fitodisponibilidade de metais pesados às culturas da soja e aveia-preta foi nula, quando foram aplicadas doses de até $8 \mathrm{Mg} \mathrm{ha}^{-1}$ de lodo de esgoto, escória e lama cal sobre a superfície do solo, no sistema plantio direto.

Termos para indexação: CTC, matéria orgânica, $\mathrm{pH}$, plantio direto.

\section{Heavy metal exchangeable in an Oxisol with surface application of flue dust, aqueous lime, sewage sludge and limestone}

\begin{abstract}
The objective of this work was to evaluate the soil chemical attributes and exchange of cadmium (Cd), chromium $(\mathrm{Cr})$, nickel $(\mathrm{Ni})$, mercury $(\mathrm{Hg})$, lead $(\mathrm{Pb})$ and arsenic $(\mathrm{As})$ by DTPA extractor in an Oxisol with surface application of flue dust, aqueous lime, sewage sludge in rates of zero (control), 2, 4 and $8 \mathrm{Mg} \mathrm{ha}^{-1}$ and one additional treatment of dolomitic limestone at $2 \mathrm{Mg} \mathrm{ha}^{-1}$. The experiment was carried out in a randomized blocks design under field conditions, in a no-tillage system in 2003 and 2004. The surface applications of flue dust, aqueous lime and sewage sludge centrifuge and biodigestor at $8 \mathrm{Mg} \mathrm{ha}^{-1}$, as well as the application of limestone at $2 \mathrm{Mg} \mathrm{ha}^{-1}$, cause no problems of exchange to the environment, related to the heavy metals $\mathrm{Cd}, \mathrm{Cr}, \mathrm{Hg}, \mathrm{Pb}, \mathrm{Ni}$ and As, when applied on an Oxisol surface in no-till system. The bioavailability of heavy metals to soybean and black-oat cultures was zero, when were applied $8 \mathrm{Mg} \mathrm{ha}^{-1}$ sewage sludge, flue dust, and aqueous lime on soil surface in no-till system.
\end{abstract}

Index terms: $\mathrm{CEC}$, organic matter, $\mathrm{pH}$, no-till.

\section{Introdução}

A utilização de resíduos industriais e de lodo de esgotos pela agricultura vem crescendo a cada ano, a fim de atender à necessidade de reciclagem; essa prática agrícola, desde que adotada de forma racional, favorece os atributos químicos, físicos e biológicos do solo (Melo et al., 2004; Nascimento et al., 2004; Corrêa et al., 2007), além de melhorar o desenvolvimento e a produtividade das espécies vegetais cultivadas (Carvalho-Puppatto et al., 2004; Nascimento et al., 2004). Deve-se ressaltar que a limitação da aplicação desses resíduos em área agrícola ocorre em razão da possível contaminação por metais pesados, que podem causar danos ao ambiente, às plantas, aos animais e ao homem (Oliveira \& Mattiazzo, 2001).

Para reduzir o risco potencial de contaminação por compostos nocivos da reação de decomposição desses resíduos ao meio, é necessário conhecer as interações entre os metais pesados e o solo, sua distribuição ao longo do perfil e sua disponibilidade à planta, pois, essa prática inspira cuidados em razão da grande velocidade de decomposição da matéria orgânica em ambiente de clima tropical e pela capacidade do solo em sorver ou 
adsorver esses metais pesados. Os metais pesados mais comuns no lodo de esgoto são chumbo $(\mathrm{Pb})$, níquel $(\mathrm{Ni})$, cádmio $(\mathrm{Cd})$, cromo $(\mathrm{Cr})$, cobre $(\mathrm{Cu})$ e zinco $(\mathrm{Zn})$ (Silveira et al., 2003).

No Estado de São Paulo, o método de extração mais estudado para a previsão da disponibilidade dos metais pesados no solo é o que emprega os agentes quelantes DTPA, entretanto, para os elementos com maior potencial tóxico como $\mathrm{Cd}, \mathrm{Cu}, \mathrm{Ni}$ e $\mathrm{Pb}$, a eficiência desse extrator tem se mostrado reduzida. É difícil haver um extrator que tenha boa correlação com as quantidades fitodisponíveis para todos os metais pesados no solo (Mattiazzo et al., 2001), no entanto, o DTPA pode ser mais eficiente que os extratores Mehlich 1 e 3 para a avaliação da disponibilidade desses elementos tóxicos no solo (Mantovani et al., 2004).

Existe a possibilidade de os metais pesados serem lixiviados em solos onde foram aplicadas altas doses de resíduos (Bertoncini \& Mattiazzo, 1999). Assim, a destinação correta desses resíduos é necessária, para se evitar poluição e contaminação ambiental, e devem ser feitas previsões de seu comportamento no solo, como os riscos de entrada de metais pesados, tendo-se como preocupação mais freqüente a solubilidade. No entanto, a redistribuição de metais no solo, que passam das formas mais lábeis para as mais estáveis, depende da espécie do metal, das propriedades do solo $(\mathrm{pH}, \mathrm{CTC}$ e matéria orgânica), do teor de metal e do tempo de incubação (McBride, 1995).

Entre os atributos químicos do solo, a elevação do $\mathrm{pH}$ favorece a adsorção dos metais pesados, interfere diretamente no deslocamento desses elementos no perfil do solo e, conseqüentemente, na absorção pela planta (Marques et al., 2001). Em solos intemperizados, onde a maior parte das cargas negativas é dependente do $\mathrm{pH}$, e onde a matéria orgânica contribui em alguns casos com aproximadamente $80 \%$ da CTC do solo (Quaggio, 2000), o aumento do $\mathrm{pH}$ no solo elevará os níveis de CTC, além de favorecer a formação de complexos e quelatos, obtidos pela matéria orgânica, fato que minimiza a disponibilidade dos metais pesados às plantas e diminui sua mobilidade no solo (Silveira et al., 2003).
Como existem alguns resíduos que apresentam a capacidade de corrigir a acidez dos solos, como: escória de aciaria, lama cal e lodo de esgoto de caráter alcalino, que podem ser utilizados em substituição ao calcário na neutralização do hidrogeniônico, além de melhorar a CTC e a matéria orgânica dos solos (Corrêa et al., 2007), suas aplicações superficiais no sistema plantio direto, possivelmente, atuarão na interação entre os metais pesados presentes nos resíduos e o solo, normalmente em suas adsorções.

O objetivo deste trabalho foi avaliar alguns atributos químicos do solo e a disponibilidade de $\mathrm{Cd}, \mathrm{Cr}, \mathrm{Ni}, \mathrm{Hg}$, $\mathrm{Pb}$ e As, por meio da extração pelo DTPA, e sua fitodisponibilidade em aveia-preta e soja, em conseqüência da aplicação superficial de escória de aciaria, lama cal e lodo de esgoto centrifugado e de biodigestor.

\section{Material e Métodos}

$\mathrm{O}$ experimento foi desenvolvido durante os anos agrícolas de 2002 a 2004, na Fazenda Experimental Lageado, da Faculdade de Ciências Agronômicas da Unesp, Botucatu, SP, a 22051'15"S, 48 $26^{\circ} 30^{\prime \prime} \mathrm{W}$ e altitude de $740 \mathrm{~m}$. Segundo a classificação climática de Köppen, o clima predominante é do tipo Cwb.

Antes da instalação do trabalho, a área experimental já havia sido conduzida no sistema de plantio direto, com a seguinte seqüência de culturas: soja, aveia, milho e um ano em pousio. Em maio de 2002, houve a dessecação da vegetação local e a posterior semeadura do feijãoguandu (Cajanus cajan), sem adubação de semeadura e de cobertura e, em agosto, aos 80 dias após a emergência desse adubo verde, realizou-se sua dessecação e a posterior montagem do experimento.

O trabalho foi implantado sob sistema de plantio direto, em Latossolo Vermelho distrófico, de textura média, fase campo subtropical, relevo suave ondulado. A amostragem da caracterização química do solo foi realizada em 15 pontos aleatórios da área (subamostra), para compor uma amostra composta de solo (Tabela 1), às profundidades 0-5 e 5-10 cm, de acordo com Raij et al. (2001).

Tabela 1. Caracterização química original do Latossolo Vermelho distrófico, em diferentes profundidades, utilizado como base experimental.

\begin{tabular}{|c|c|c|c|c|c|c|c|c|c|c|c|c|}
\hline $\begin{array}{l}\text { Profundidade } \\
(\mathrm{cm})\end{array}$ & $\underset{\mathrm{CaCl}_{2}}{\mathrm{pH}}$ & $\begin{array}{c}\mathrm{MO} \\
\left(\mathrm{g} \mathrm{dm}^{-3}\right)\end{array}$ & $\begin{array}{l}\text { P-resina } \\
\left(\mathrm{mg} \mathrm{dm}^{-3}\right)\end{array}$ & $\mathrm{Al}^{3+}$ & $\mathrm{H}+\mathrm{Al}$ & $\mathrm{K}$ & $\begin{array}{r}\mathrm{Ca} \\
\left(\mathrm{mmol}_{\mathrm{c}} \mathrm{C}\right.\end{array}$ & $\mathrm{Mg}$ & SB & CTC & $\begin{array}{l}\mathrm{V} \\
(\%)\end{array}$ & $\begin{array}{c}\mathrm{m} \\
(\%)\end{array}$ \\
\hline $0-5$ & 4,0 & 16 & 6 & 7 & 38 & 0,6 & 12 & 6 & 19 & 57 & 33 & 12 \\
\hline $5-10$ & 4,0 & 18 & 6 & 6 & 45 & 1,0 & 19 & 9 & 29 & 74 & 39 & 5 \\
\hline
\end{tabular}


Os tratamentos se constituíram da utilização de quatro resíduos, dos quais: dois tipos de lodo de esgoto, um centrifugado com adição de cal virgem (LC), da Estação de Tratamento de Esgoto (ETE) de São José dos Campos, SP, e o segundo foi lodo de biodigestor com adição de polieletrólitos (LB), da ETE de Barueri, $\mathrm{SP}$; e dois resíduos industriais, que foram escória de aciaria da Empresa Mannesmann (E), e lama cal, da Empresa de celulose Luwarcel (Lcal), cuja caracterização química está apresentada na Tabela 2. Foram empregadas as doses equivalentes a 0 (testemunha), 2, 4 e $8 \mathrm{Mg} \mathrm{ha}^{-1}$ para cada resíduo. Foi utilizado, também, um tratamento-controle (padrão), que correspondeu à aplicação de calcário dolomítico (280 $\mathrm{g} \mathrm{kg}^{-1}$ de $\mathrm{CaO}, 230 \mathrm{~g} \mathrm{~kg}^{-1}$ de $\mathrm{MgO}$ e PRNT de $71,29 \%$ ), na dose recomendada pelo método de saturação por bases $\left(2 \mathrm{Mg} \mathrm{ha}^{-1}\right)$ para elevar este índice a $60 \%$, com base de cálculo para a profundidade de $0-20 \mathrm{~cm}$ para produção de soja (Raij et al., 1996).

Antes da instalação do experimento, determinou-se a quantidade total de metais pesados presentes em cada resíduo (Tabela 2), conforme Laboratório Nacional de Referência Vegetal (1983). As leituras foram analisadas em espectrofotômetro de emissão em plasma, induzida em argônio (ICP/AES), tendo-se comparado os valores dos resíduos industriais Lcal e $\mathrm{E}$ às quantidades permitidas na norma de aplicação de resíduos sólidos, conforme a NBR 10004 (Associação Brasileira de Normas Técnicas, 1987) e os valores de lodo de esgoto - LB e LC - à legislação imposta pela Cetesb (1999) na Norma P4230. Vale ressaltar que os quatro resíduos apresentaram resultados inferiores aos valores limítrofes propostos pela Legislação Brasileira.

Foram determinados: a reatividade (RE), o poder neutralizante $(\mathrm{PN})$ e o poder relativo de neutralização total (PRNT) da lama cal, do lodo de esgoto (LC e LB), da escória de aciaria, e do calcário conforme Laboratório Nacional de Referência Vegetal (1983) cujos resultados estão apresentados na Tabela 3, o que torna possível a comparação entre os tratamentos como materiais corretivos da acidez.

Os tratamentos foram distribuídos no campo, em delineamento de blocos ao acaso, com esquema fatorial $4 \times 4+1$, com quatro repetições. Em cada bloco, as parcelas foram de $6 \mathrm{~m}$ de largura por $7 \mathrm{~m}$ de comprimento, tendo-se deixado $1 \mathrm{~m}$ entre as parcelas do mesmo bloco e $3 \mathrm{~m}$ entre blocos.

Nas safras 2002/2003 e 2003/2004, foi cultivada soja (Glycine $\max$ L.), tendo-se aplicado adubação de $300 \mathrm{~kg} \mathrm{ha}^{-1}$ com o fertilizante formulado 0-20-10, com sementes com inoculação de Bradyrhizobium japonicum. Na entressafra, nos anos de 2003 e 2004, foi cultivada aveia-preta (Avena strigosa Schreb.), tendo-se aplicado adubação nitrogenada $\left(100 \mathrm{~kg} \mathrm{ha}^{-1} \mathrm{de}\right.$ uréia), com a finalidade de produção de massa verde e, posteriormente, palhada. Para as quatro culturas instaladas na área, entre 2002 a 2004, foi adotado o sistema plantio direto.

Tabela 2. Análise química dos resíduos industriais e urbanos, utilizados como tratamentos no experimento sob plantio direto.

\begin{tabular}{|c|c|c|c|c|c|c|c|c|c|c|}
\hline Resíduo & Umidade & $\mathrm{MO}$ & $\mathrm{pH}$ & $\mathrm{S}$ & As & $\mathrm{Cd}$ & $\begin{array}{c}\mathrm{Cr} \\
\mathrm{m}^{-3} \mathrm{de}\end{array}$ & $\mathrm{Hg}$ & $\mathrm{Ni}$ & $\mathrm{Pb}$ \\
\hline $\mathrm{LC}$ & 5 & 26 & 12 & 0,5 & 14,0 & tracos & 4 & tracos & 7 & 17 \\
\hline LB & 29 & 50 & 7 & 1,6 & 27,0 & 0,1 & 19 & traços & 180 & 107 \\
\hline Lcal & 19 & 3 & 12 & 0,3 & 1,4 & traços & 13 & traços & 96 & 60 \\
\hline $\mathrm{E}$ & 2 & 1 & 12 & - & 5,0 & traços & 61 & traços & 19 & 308 \\
\hline $\bar{C}$ etesb $1999^{(1)}$ & & & & & 75,0 & 85 & 3.000 & 57 & 420 & 840 \\
\hline NBR $10004^{(2)}$ & & & & & 1.000 & n.a. ${ }^{(3)}$ & n.a. & 100 & n.a. & 1.000 \\
\hline
\end{tabular}

${ }^{(1)}$ Legislação para os limites máximos de metais pesados permitidos em lodos de esgoto, imposta pela Cetesb (1999), Lei P4230. (2)Legislação para os limites máximos de metais pesados permitidos em resíduos sólidos, imposta pela Associação Brasileira de Normas Técnicas (1987), Lei NBR 10004. (3)Não aplicável (não foi descrito o limite na NBR 10004).

Tabela 3. Resultados em percentagem de partículas retidas nas peneiras de $10(\varnothing=2 \mathrm{~mm}), 20(\varnothing=0,84 \mathrm{~mm}), 50(\varnothing=0,30 \mathrm{~mm})$ e fundo, e reatividade (RE), poder neutralizante (PN) e poder relativo de neutralização total (PRNT).

\begin{tabular}{|c|c|c|c|c|c|c|c|}
\hline Resíduo & Pen 10 & Pen 20 & Pen 50 & Fundo & $\mathrm{RE}$ & $\mathrm{PN}$ & PRNT \\
\hline Lcal & 0,00 & 0,00 & 0,05 & 99,95 & 99,98 & 99,00 & 98,98 \\
\hline LC & 2,40 & 14,63 & 40,10 & 42,87 & 69,85 & 93,00 & 65,00 \\
\hline $\mathrm{E}$ & 2,20 & 6,15 & 32,53 & 59,10 & 79,80 & 60,00 & 47,88 \\
\hline LB & 0,09 & 50,23 & 35,46 & 14,22 & 36,50 & 3,00 & 1,09 \\
\hline Calcário & 0,00 & 4,20 & 18,80 & 77,00 & 89,12 & 80,00 & 71,29 \\
\hline
\end{tabular}


$\mathrm{Na}$ amostragem de solo, às profundidades de $0-5 \mathrm{e}$ 5-10 cm, foi utilizado um trado do tipo sonda, para coletar quatro amostras por parcela, que constituíram uma amostra composta, todos anteriores à semeadura da soja nos anos de 2003 e 2004. Foram realizadas as seguintes análises químicas: $\mathrm{pH}$ em $\mathrm{CaCl}_{2}(0,01 \mathrm{M})$, matéria orgânica e $\mathrm{K}, \mathrm{Ca}, \mathrm{Mg}$ e $\mathrm{H}+\mathrm{Al}$, para determinação da CTC do solo, além das análises dos metais pesados $\mathrm{Cd}$, $\mathrm{Cr}, \mathrm{Ni}, \mathrm{Hg}, \mathrm{Pb}$ e As, extraídos em DTPA (Raij et al., 2001).

Para as análises de metais pesados no solo, foram aproveitadas as mesmas amostras da análise química, nas mesmas profundidades, pela metodologia da extração em solução de DTPA em pH 7,3. As leituras do extrato do solo foram realizadas em espectrofotômetro de emissão em plasma induzida em argônio (ICP/AES).

Para a determinação da fitodisponibilidade de metais pesados na planta, foram coletadas 50 folhas bandeiras de aveia-preta, por parcela, e 50 trifólios de soja por parcela, o terceiro a partir do ápice, no estádio R2, em pleno florescimento. Os metais pesados foram determinados pela digestão nitro-perclórica (Malavolta et al., 1997).

Os resultados foram submetidos à análise de variância e posterior regressão, tendo-se ajustado as equações aos dados obtidos e adotado, como critério para a escolha do modelo, a interação pelo teste $\mathrm{F}$, a 1 e $5 \%$ de probabilidade e a magnitude dos coeficientes de determinação (Banzatto \& Kronka, 1989); os resultados foram analisados pelo programa Sigmaplot 8.0. Todos os tratamentos foram comparados ao controle calcário pelo teste de média DMS, a 1 e $5 \%$ de probabilidade (Banzatto \& Kronka, 1989), com o programa Sisvar 4,2. Foi realizada a correlação de Pearson, para se verificar a existência de possíveis relações entre os metais pesados e os atributos químicos do solo.

\section{Resultados e Discussão}

A aplicação superficial dos lodos de esgoto LB e LC e dos resíduos industriais E e Lcal, em plantio direto, não causou problemas de poluição ambiental, considerando-se a presença do teor disponível de metais pesados $\mathrm{Cd}, \mathrm{Cr}, \mathrm{Hg}, \mathrm{Pb}$, Ni e As no solo, nos anos de 2003 e 2004 (Tabela 4). De acordo com Mattiazzo et al. (2001), não existe extrator que tenha boa correlação com as quantidades fitodisponíveis para todos os metais pesados existentes no lodo de esgoto, e há necessidade de se padronizar os métodos de análises, a fim de se maximizarem os resultados obtidos em condições de solos ácidos. No entanto, Mantovani et al. (2004) constataram que o DTPA pode ser mais eficiente que os extratores Mehlich 1 e 3, para a avaliação da fitodisponibilidade de metais pesados.

No ano de 2003, com a aplicação superficial dos resíduos no solo, foram encontrados traços de $\mathrm{Cd}, \mathrm{Cr}$, $\mathrm{Ni}$ e $\mathrm{Hg}$ no solo, e foram detectados apenas $\mathrm{As}$ e $\mathrm{Pb}$, sem diferença em consequiência do aumento das doses dos resíduos (Tabela 4). Assim, a adição de $8 \mathrm{Mg} \mathrm{ha}^{-1}$ de resíduos, aplicados sobre a superfície do solo, não diferem dos teores disponíveis de metais pesados onde esses não foram aplicados (dose zero), no ano de 2003.

No ano de 2004, não houve aumento nos teores de As, $\mathrm{Cr}, \mathrm{Hg}$ e $\mathrm{Pb}$, nas camadas de $0-5$ e $5-10 \mathrm{~cm}$ de profundidade, o que demonstra que as doses aplicadas de lodo de esgoto e de resíduos industriais utilizadas não aumentaram a disponibilidade desses metais pesados no solo, o que possibilita a aplicação racional desses mesmos metais na agricultura. A aplicação de LB e E influenciou significativamente os teores de $\mathrm{Cd}$ no solo. Na camada $0-5 \mathrm{~cm}$, houve efeito quadrático, em que o teor de $\mathrm{Cd}$ atingiu o máximo de $17,01 \mu \mathrm{g} \mathrm{dm}^{-3} \mathrm{com}$ a dose de 6,6 $\mathrm{Mg} \mathrm{ha}^{-1} \mathrm{de} \mathrm{LB}$, e de $8,08 \mu \mathrm{g} \mathrm{dm}^{-3}$ com a dose de $3,03 \mathrm{Mg} \mathrm{ha}^{-1}$ de E. Na camada 5-10 cm, LB e E tiveram comportamento linear crescente, em que LB apresentou maiores teores em relação ao $\mathrm{E}$. Os valores de $\mathrm{Cd}$ encontrados na camada $0-5 \mathrm{~cm}$ foram maiores que os da camada $5-10 \mathrm{~cm}$, tanto para LB como para E. $\mathrm{O}$ tratamento $\mathrm{LB}$ foi o único que apresentou $\mathrm{Cd}$ em sua composição (Tabela 2), e este fato explica os resultados encontrados. Quanto a E, por se tratar de resíduo de siderurgia, é provável que o $\mathrm{Cd}$, mesmo em pequenas concentrações em sua composição (traço), favoreça o aumento dos teores disponíveis deste metal pesado, quando aplicado em grandes quantidades.

A aplicação do LB reduziu os teores disponíveis de $\mathrm{Ni}$, em conseqüência do aumento das doses, para as camadas $0-5$ e $5-10 \mathrm{~cm}$ de profundidade. Isto pode ser explicado pela presença de 50\% de matéria orgânica em sua composição, que produz complexos e quelatos que podem fixar o $\mathrm{Ni}$, o que contribui para diminuir sua disponibilidade no solo. Vale lembrar que, pelo fato de haver $180 \mathrm{mg} \mathrm{dm}^{-3}$ na matéria seca do LB, é possível que o teor total do Ni no solo tenha aumentado.

Os resultados obtidos concordam com os de Nascimento et al. (2004), o que demonstra que a aplicação de lodo de esgoto pode ser realizada sem maiores riscos de poluição com metais pesados, e 
corroboram, também, os resultados de Andreoli \& Pegorini (2000), que informam que o uso agrícola do lodo de esgoto foi exaustivamente pesquisado em todo o mundo, não tendo havido registro de nenhum efeito adverso sobre o ambiente, quando foram observadas as regulamentações existentes sobre o uso como insumo agrícola.

A aplicação superficial de doses crescentes de LC, Lcal e E proporcionaram o aumento de $\mathrm{pH}$ no solo (Figura 1), e em conjunto com o LB elevam os teores

Tabela 4. Disponibilidade de metais pesados no solo nos anos de 2003 e 2004, extraídos por DTPA, pela aplicação superficial de doses crescentes de escória de aciaria, lama cal, lodos de esgoto e calcário em plantio direto.

\begin{tabular}{|c|c|c|c|c|c|c|c|c|c|}
\hline \multirow[t]{2}{*}{ Resíduo } & \multirow[t]{2}{*}{ Dose $\left(\mathrm{t} \mathrm{ha}^{-1}\right)$} & \multicolumn{2}{|c|}{2003} & \multicolumn{6}{|c|}{2004} \\
\hline & & As & $\overline{\mathrm{Pb}}$ & $\mathrm{Cd}$ & $\mathrm{Cr}$ & $\mathrm{Hg}$ & $\mathrm{Ni}$ & $\mathrm{Pb}$ & $\mathrm{V}$ \\
\hline & & \multicolumn{8}{|c|}{ Camada $0-5 \mathrm{~cm}$} \\
\hline $\mathrm{LC}$ & 0 & 0,003 & 0,06 & 0,005 & 0,32 & 0,007 & 0,21 & 0,02 & 0,03 \\
\hline $\mathrm{LC}$ & 2 & 0,003 & 0,04 & 0,005 & 0,31 & 0,010 & 0,22 & 0,01 & 0,02 \\
\hline $\mathrm{LC}$ & 4 & 0,004 & 0,05 & 0,008 & 0,32 & 0,010 & 0,22 & 0,00 & 0,03 \\
\hline $\mathrm{LC}$ & 8 & 0,003 & 0,03 & 0,007 & 0,31 & 0,010 & 0,22 & 0,01 & 0,03 \\
\hline Média & & 0,004 & 0,05 & 0,006 & 0,32 & 0,009 & 0,22 & 0,01 & 0,03 \\
\hline LB & 0 & 0,004 & 0,06 & 0,005 & 0,32 & 0,007 & 0,21 & 0,02 & 0,03 \\
\hline LB & 2 & 0,007 & 0,07 & 0,015 & 0,32 & 0,011 & 0,13 & 0,03 & 0,03 \\
\hline LB & 4 & 0,005 & 0,08 & 0,013 & 0,32 & 0,009 & 0,14 & 0,02 & 0,02 \\
\hline LB & 8 & 0,011 & 0,11 & 0,017 & 0,31 & 0,009 & 0,08 & 0,02 & 0,02 \\
\hline Média & & 0,007 & 0,08 & 0,012 & 0,32 & 0,009 & 0,14 & 0,02 & 0,03 \\
\hline Regressão & & ns & ns & $\mathrm{Q}^{* *}$ & $\mathrm{~ns}$ & ns & $\mathrm{L}^{* *}$ & ns & $\mathrm{ns}$ \\
\hline Lcal & 0 & 0,003 & 0,06 & 0,005 & 0,32 & 0,007 & 0,21 & 0,02 & 0,03 \\
\hline Lcal & 2 & 0,006 & 0,06 & 0,004 & 0,32 & 0,010 & 0,22 & 0,01 & 0,03 \\
\hline Lcal & 8 & 0,001 & 0,07 & 0,005 & 0,32 & 0,009 & 0,22 & 0,01 & 0,03 \\
\hline Média & & 0,003 & 0,05 & 0,005 & 0,32 & 0,008 & 0,22 & 0,01 & 0,03 \\
\hline Regressão & & ns & ns & $\mathrm{ns}$ & ns & ns & ns & ns & ns \\
\hline $\mathrm{E}$ & 0 & 0,003 & 0,06 & 0,005 & 0,32 & 0,007 & 0,21 & 0,02 & 0,03 \\
\hline $\mathrm{E}$ & 2 & 0,004 & 0,04 & 0,009 & 0,31 & 0,011 & 0,21 & 0,03 & 0,02 \\
\hline $\mathrm{E}$ & 4 & 0,002 & 0,04 & 0,007 & 0,31 & 0,009 & 0,21 & 0,01 & 0,02 \\
\hline E & 8 & 0,002 & 0,05 & 0,003 & 0,31 & 0,008 & 0,22 & 0,01 & 0,02 \\
\hline Média & & 0,003 & 0,05 & 0,006 & 0,32 & 0,009 & 0,21 & 0,02 & 0,02 \\
\hline Regressão & & ns & ns & $\mathrm{Q}^{* *}$ & ns & ns & ns & ns & ns \\
\hline \multicolumn{2}{|l|}{ Calcário } & 0,006 & 0,015 & 0,004 & 0,31 & 0,011 & 0,22 & 0,02 & 0,02 \\
\hline & & \multicolumn{8}{|c|}{ Camada $5-10 \mathrm{~cm}$} \\
\hline $\mathrm{LC}$ & 0 & 0,003 & 0,06 & 0,002 & 0,32 & 0,009 & 0,21 & 0,01 & 0,03 \\
\hline $\mathrm{LC}$ & 2 & 0,004 & 0,04 & 0,003 & 0,31 & 0,070 & 0,21 & 0,02 & 0,02 \\
\hline $\mathrm{LC}$ & 4 & 0,004 & 0,05 & 0,004 & 0,32 & 0,100 & 0,22 & 0,01 & 0,03 \\
\hline $\mathrm{LC}$ & 8 & 0,001 & 0,04 & 0,003 & 0,31 & 0,098 & 0,21 & 0,01 & 0,02 \\
\hline Média & & 0,003 & 0,05 & 0,003 & 0,31 & 0,100 & 0,21 & 0,01 & 0,03 \\
\hline Regressão & & $\mathrm{ns}$ & ns & $\underset{n s}{n}$ & ns & $\mathrm{ns}$ & $\mathrm{ns}$ & ns & ns \\
\hline LB & 4 & 0,005 & 0,05 & 0,004 & 0,31 & 0,110 & 0,20 & 0,03 & 0,03 \\
\hline LB & 8 & 0,009 & 0,07 & 0,005 & 0,31 & 0,088 & 0,16 & 0,02 & 0,03 \\
\hline Média & & 0,006 & 0,07 & 0,004 & 0,31 & 0,085 & 0,19 & 0,02 & 0,03 \\
\hline Regressão & & ns & ns & $\mathrm{L}^{* *}$ & ns & & $\mathrm{L} * *$ & ns & $\mathrm{ns}$ \\
\hline Lcal & 0 & 0,003 & 0,06 & 0,002 & 0,32 & 0,009 & 0,21 & 0,01 & 0,03 \\
\hline Lcal & 2 & 0,005 & 0,08 & 0,004 & 0,32 & 0,070 & 0,21 & 0,02 & 0,02 \\
\hline Lcal & 4 & 0,004 & 0,05 & 0,002 & 0,31 & 0,095 & 0,22 & 0,02 & 0,02 \\
\hline Lcal & 8 & 0,001 & 0,05 & 0,003 & 0,31 & 0,078 & 0,21 & 0,01 & 0,02 \\
\hline Média & & 0,003 & 0,06 & 0,003 & 0,32 & 0,093 & 0,21 & 0,02 & 0,02 \\
\hline Regressão & & ns & ns & $\begin{array}{c}0,00 J \\
n s\end{array}$ & ns & ns & ns & ns & ns \\
\hline $\mathrm{E}$ & 0 & 0,003 & 0,06 & 0,002 & 0,32 & 0,009 & 0,21 & 0,01 & 0,03 \\
\hline $\mathrm{E}$ & 2 & 0,002 & 0,05 & 0,003 & 0,32 & 0,070 & 0,21 & 0,04 & 0,03 \\
\hline $\mathrm{E}$ & 4 & 0,001 & 0,07 & 0,003 & 0,31 & 0,108 & 0,21 & 0,01 & 0,02 \\
\hline E & 8 & 0,001 & 0,08 & 0,004 & 0,31 & 0,090 & 0,21 & 0,01 & 0,03 \\
\hline Média & & 0,002 & 0,07 & 0,003 & 0,31 & 0,077 & 0,21 & 0,02 & 0,02 \\
\hline$\underline{\text { Regressão }}$ & & ns & ns & $\mathrm{L} * *$ & ns & $\mathrm{ns}$ & $\mathrm{ns}$ & ns & ns \\
\hline Calcário & & 0,005 & 0,01 & 0,003 & 0,31 & 0,020 & 0,22 & 0,02 & 0,02 \\
\hline
\end{tabular}

(1)LC: lodo de esgoto centrifugado; LN: lodo de esgoto de biodigestor; E: escória de aciária; Lcal: lama cal; Regressão: L: linear e Q: quadrática.

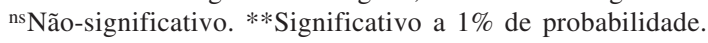


de matéria orgânica e CTC do solo (Figuras 2 e 3), tanto em 2003 como em 2004. Os metais pesados apresentaram elevada afinidade com os colóides do solo, e foram adsorvidos através de ligações covalentes, em razão da presença de cargas negativas do solo (Marques et al., 2001; Borges \& Coutinho, 2004). Assim, nos solos intemperizados, em que a maior parte das cargas negativas é dependente do $\mathrm{pH}$, e a matéria orgânica contribui em alguns casos com aproximadamente $80 \%$ da CTC do solo (Quaggio, 2000), o aumento do $\mathrm{pH}$ no solo elevará os níveis de CTC, além de favorecer a formação de complexos e quelatos, obtidos pela matéria orgânica, fato que minimiza a disponibilidade dos metais pesados às plantas e diminui sua mobilidade no solo (Silveira et al., 2003).

Com o aumento do pH do solo pela aplicação superficial dos resíduos E, Lcal e LC, além do tratamento calcário (Figura 1), é possível diminuir a disponibilidade dos metais pesados no solo, interferir diretamente em seu deslocamento no perfil do solo e, conseqüentemente, interferir na absorção pelas plantas (Marques et al., 2001). Em solos intemperizados, como o Latossolo Vermelho distrófico, a presença de colóides com cargas dependentes de $\mathrm{pH}$ chegam a representar mais de $70 \%$ do total de pontos de ligação de cátions. Assim, nesses solos, torna-se indispensável a correção da acidez para a maior adsorção dos metais pesados.

$\mathrm{O}$ aumento no teor da matéria orgânica do solo, pela aplicação superficial de doses crescentes dos resíduos e do calcário (Figura 2), somado à presença da matéria orgânica do lodo ainda não decomposta nos anos de 2003 e 2004, favorece a adsorção do metais pesados a essa fração do solo, em razão da formação de complexos ou
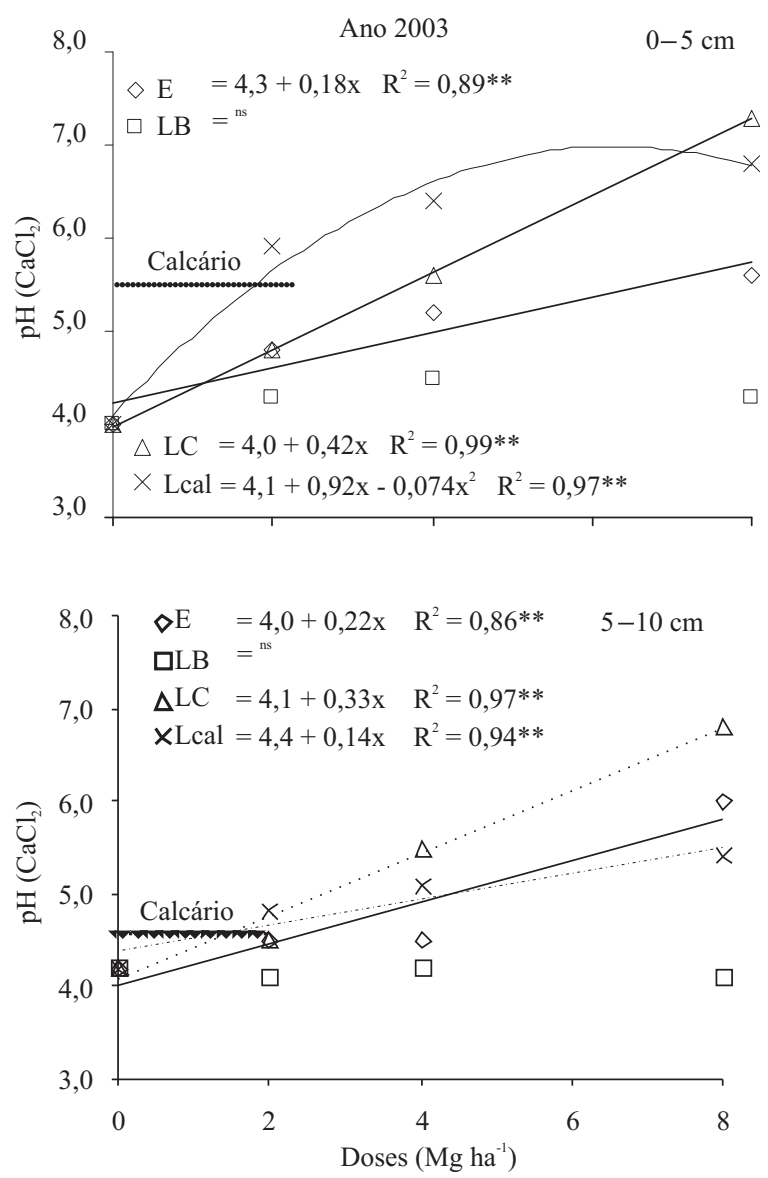

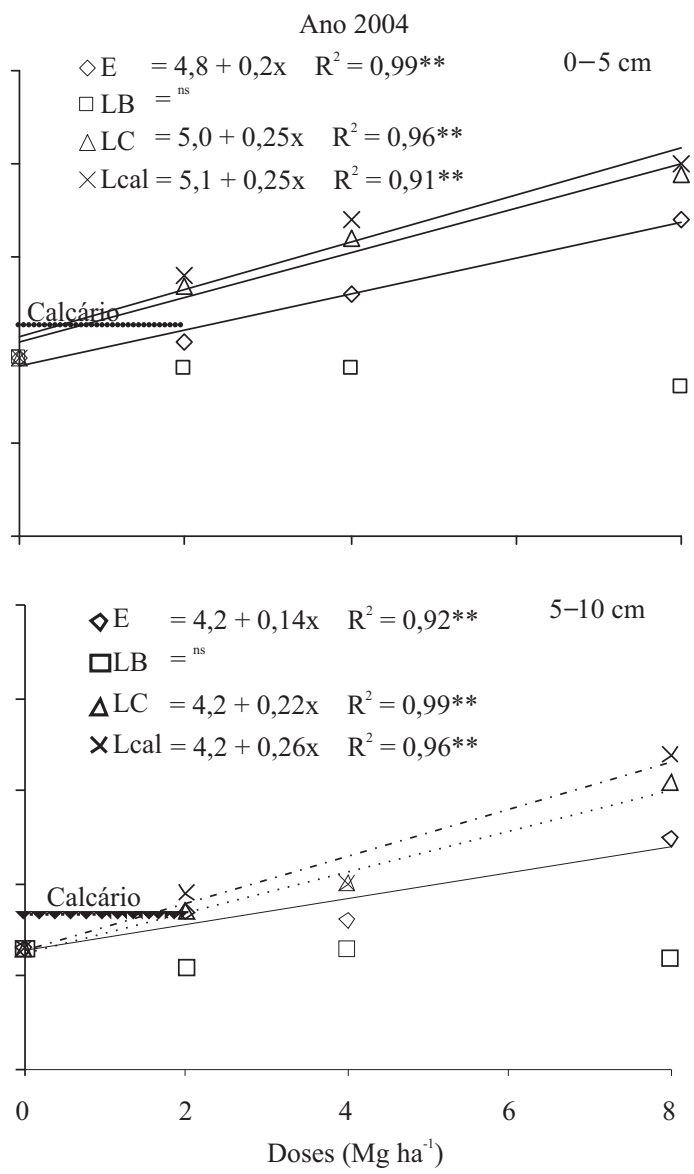

Figura 1. Valores de $\mathrm{pH}$ em $\mathrm{CaCl}_{2}(0,01 \mathrm{M})$ no solo, em conseqüência da aplicação superficial de doses crescentes de escória de

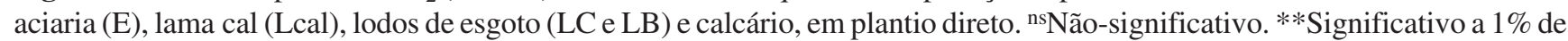
probabilidade. 
pela quelatização (Marques et al., 2001). Borges \& Coutinho (2004) demonstraram que a aplicação de lodo de esgoto diminui a disponibilidade do $\mathrm{Pb}$ às plantas, pelo fato de esse elemento passar da forma trocável para as frações menos fitodisponíveis, pela maior presença da forma orgânica.

A aplicação superficial dos resíduos e do calcário em sistema plantio direto elevou os teores de CTC no solo (Figura 3), o que possibilitou maior adsorção dos metais pesados catiônicos pelos colóides do solo, e a ligação do tipo covalente aos colóides, o que ajuda a explicar a baixa disponibilidade de metais pesados no solo (Tabela 4). Nos solos intemperizados, o aumento da CTC está associado ao aumento de $\mathrm{pH}$, em razão da maior quantidade de cargas negativas dependentes de
$\mathrm{pH}$, caracterizada pela maior presença de minerais do tipo 1:1 e de óxidos hidratados de Fe e Al. Vale ressaltar a adsorção de $\mathrm{Cd}^{2+}, \mathrm{Cu}^{2+} \mathrm{Pb}^{2+} \mathrm{e} \mathrm{Zn}^{2+}$, realizada pelos óxidos de ferro sob forte energia de ligação, que pode formar estruturas sólidas como plintita (Martinez \& McBride, 1998).

Não houve correlação significativa entre os teores disponíveis de $\mathrm{Cd}, \mathrm{Cr}, \mathrm{Hg}, \mathrm{Pb}, \mathrm{V}$ e As, em relação aos atributos químicos do solo - $\mathrm{pH}$, matéria orgânica e CTC -, o que permite inferir que houve efeito de compensação entre a adição de metais pesados pelos resíduos e a sua atuação no aumento do $\mathrm{pH}$, da matéria orgânica e da CTC do solo (responsáveis pela adsorção desses metais), o que os torna indisponíveis para análise de extração por DTPA, assim como indisponíveis às plantas, nas condições de Latossolo Vermelho distrófico, manejado sob plantio direto.
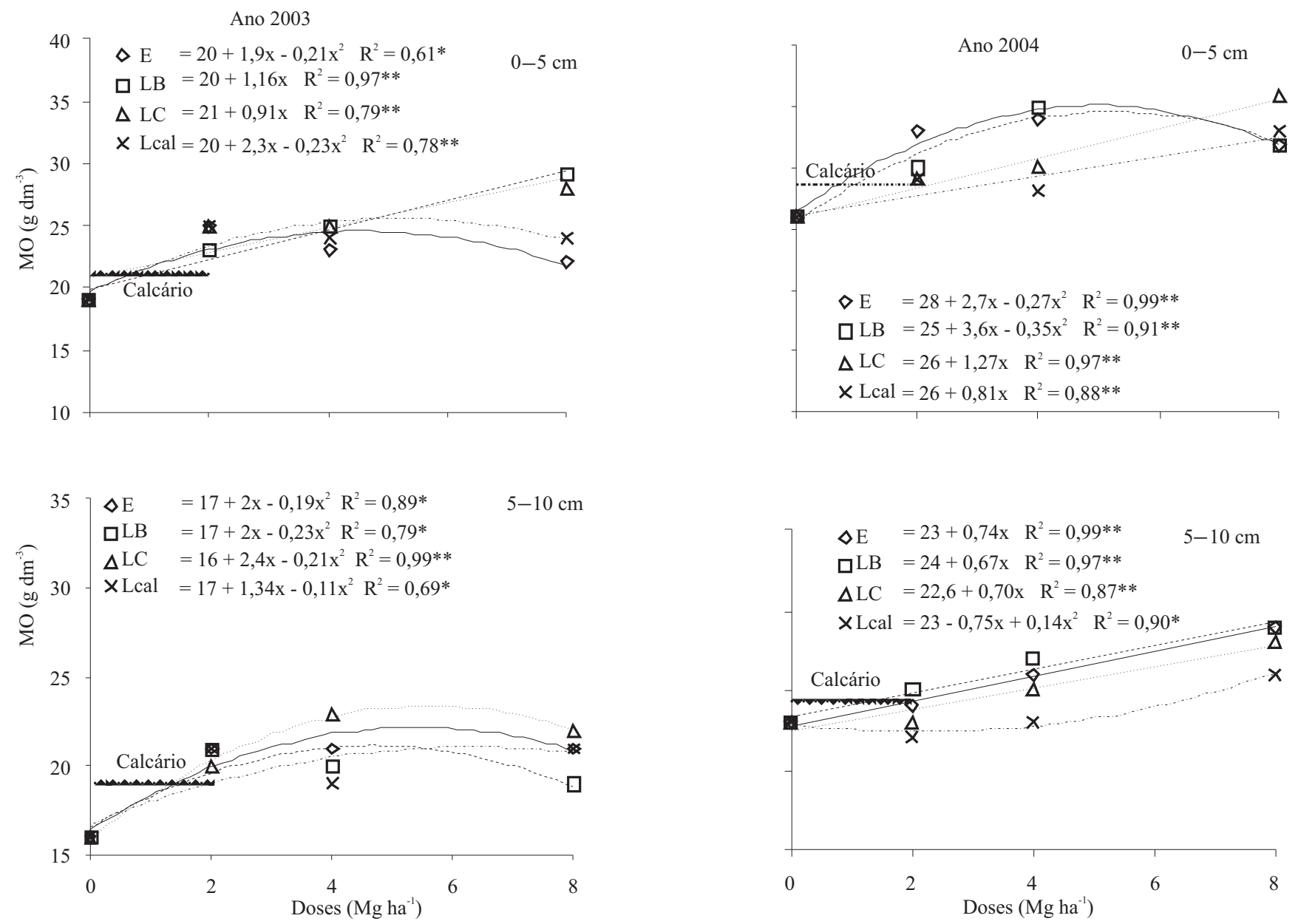

Figura 2. Teores de matéria orgânica no solo, em conseqüência da aplicação superficial de doses crescentes de escória de aciaria (E), lama cal (Lcal), lodos de esgoto (LC e LB) e calcário, em plantio direto. * e **Significativo a 5 e $1 \%$ de probabilidade, respectivamente. 

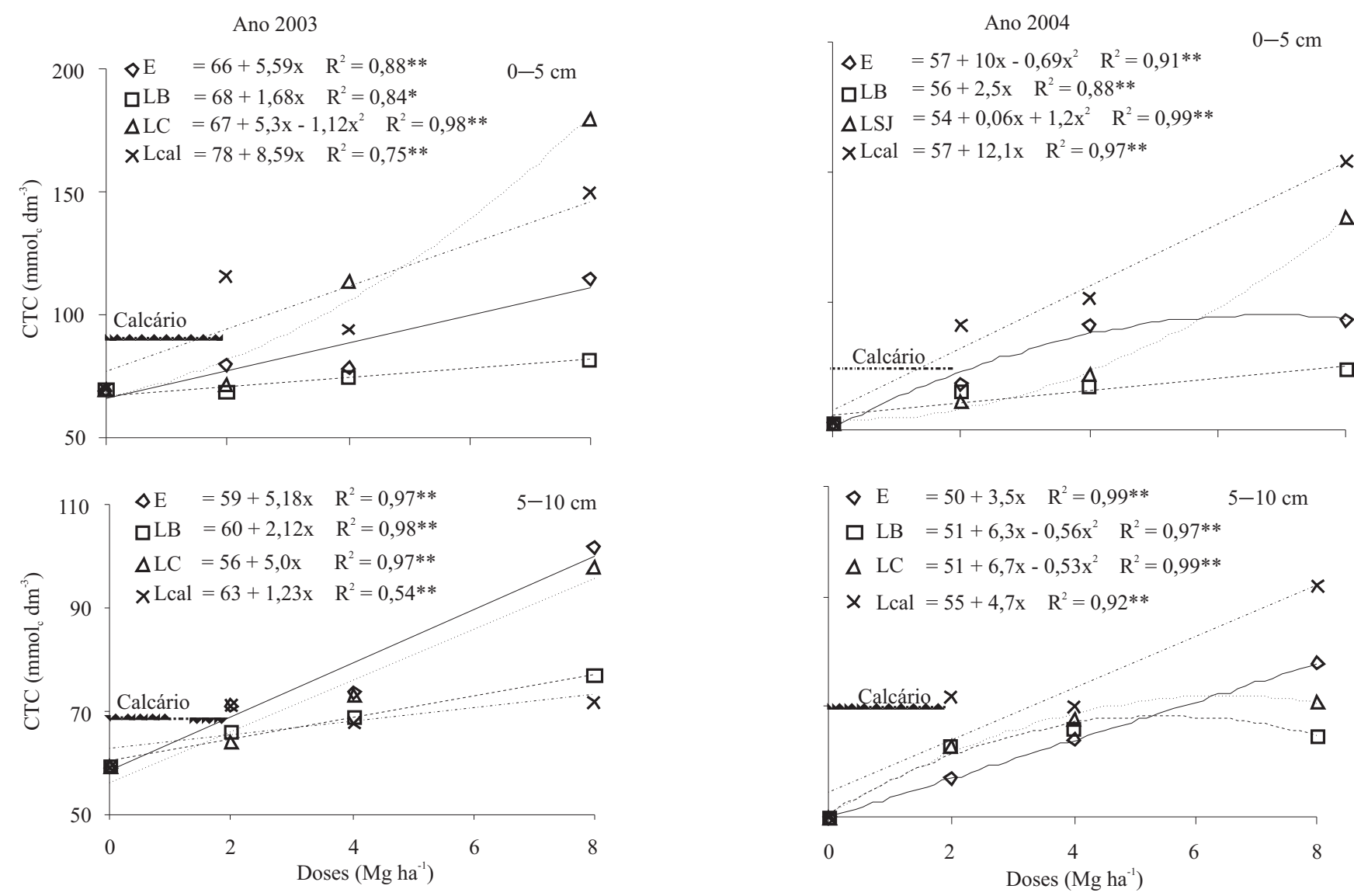

Figura 3. Capacidade de troca de cátions do solo, em consequiência da aplicação superficial de doses crescentes de escória de aciaria (E), lama cal (Lcal), lodos de esgoto (LC e LB) e calcário, em plantio direto. * e**Significativo a 5 e $1 \%$ de probabilidade, respectivamente.

Em razão da baixíssima disponibilidade dos metais pesados presentes no solo $(\mathrm{Cd}, \mathrm{Cr}, \mathrm{Ni}, \mathrm{Hg}, \mathrm{Pb}$ e $\mathrm{As})$, não houve a fitodisponibilidade desses elementos tóxicos às culturas de soja e de aveia-preta, tendo sido detectados apenas traços no teor desses metais na folhas-diagnóstico das culturas. De acordo com esses resultados, doses de até $8 \mathrm{Mg} \mathrm{ha}^{-1}$ dos lodos de esgoto de biodigestor e centrifugado e dos resíduos industriais escória e lama cal são passíveis de serem aplicadas ao solo, sem causar problemas de fitotoxicidade a essas culturas.

$\mathrm{O}$ aumento das concentrações de metais pesados no solo, resultante da aplicação sucessiva de lodo de esgoto, pode, a longo prazo, tornar-se uma preocupação justificada (López-Mosquera et al., 2000), pois se não for adequadamente controlado, esse aumento pode ameaçar a cadeia trófica (Hue, 1995). Então, pode-se recomendar o uso agrícola dos resíduos LC, LB, Lcal e E, em superfície sob plantio direto, até a dose de $8 \mathrm{Mg} \mathrm{ha}^{-1}$, uma única vez, sem prejuízo de contaminação ambiental, nas condições de Latossolo Vermelho distrófico, e em condições climáticas do tipo Cwa com aproximadamente $1.500 \mathrm{~mm}$ de chuva por ano, sob constante monitoramento da matéria orgânica do solo, da disponibilidade no solo e da fitodisponibilidade na planta, dos metais pesados $\mathrm{Cd}, \mathrm{Cr}$, $\mathrm{Hg}, \mathrm{Pb}, \mathrm{Ni}$ e As.

\section{Conclusões}

1. A aplicação superficial de escória de aciaria, lama cal, lodo de esgoto centrifugado e de biodigestor, até a dose de $8 \mathrm{Mg} \mathrm{ha}^{-1}$, não aumenta a disponibilidade no solo dos metais pesados $\mathrm{Cd}, \mathrm{Cr}, \mathrm{Hg}, \mathrm{Pb}$, Ni e As, extraídos pelo DTPA, quando são aplicados na superfície de Latossolo Vermelho distrófico, em sistema plantio direto.

2. A fitodisponibilidade dos metais pesados $\mathrm{Cd}, \mathrm{Cr}$, $\mathrm{Hg}, \mathrm{Pb}, \mathrm{Ni}$ e As às culturas da soja e aveia-preta é nula, quando são aplicadas doses de até $8 \mathrm{Mg} \mathrm{ha}^{-1}$ de lodos de esgoto centrifugado e de biodigestor, escória de 
aciaria e lama cal, na superfície do solo, no sistema plantio direto.

\section{Agradecimentos}

À Fundação de Amparo à Pesquisa no Estado de São Paulo, pela bolsa e pelo apoio financeiro concedidos.

\section{Referências}

ANDREOLI, C.V.; PEGORINI, E.S. Gestão pública do uso agrícola do lodo de esgoto. In: BETTIOL, W.; CAMARGO, O.A. (Ed.). Impacto ambiental do uso agrícola do lodo de esgoto. Jaguariúna: Embrapa Meio Ambiente, 2000. p.281-312.

ASSOCIAÇÃO BRASILEIRA DE NORMAS TÉCNICAS. NBR 10004: resíduos sólidos: classificação. Rio de Janeiro, 1987. 63p.

BANZATTO, D.A.; KRONKA, S.N. Experimentação agrícola. Jaboticabal: Funep, 1989. 247p.

BERTONCINI, E.I.; MATTIAZZO, M.E. Lixiviação de metais pesados em solos tratados com lodo de esgoto. Revista Brasileira de Ciência do Solo, v.23, p.737-744, 1999.

BORGES, M.R.; COUTINHO, E.L.M. Metais pesados do solo após aplicação de biossólido: II - Disponibilidade. Revista Brasileira de Ciência do Solo, v.28, p.557-568, 2004.

CARVALHO-PUPATTO, J.G.; BÜLL, L.T.; CRUSCIOL, C.A.C. Atributos químicos do solo, crescimento radicular e produtividade do arroz de acordo com a aplicação de escórias. Pesquisa Agropecuária Brasileira, v.39, p.1213-1218, 2004.

CETESB. Aplicação de lodos de sistemas de tratamento biológico em áreas agrícolas: critérios para projeto e operação. São Paulo: Cetesb, 1999. 32p. (Norma P 4230).

CORRÊA, J.C.; BÜLL, L.T.; CRUSCIOL, C.A.C.; MAUAD, M.; MARCELINO, M. Correção da acidez e mobilidade de íons em Latossolo com aplicação superficial de escória de aciaria, lama cal, lodos de esgoto e calcário. Pesquisa Agropecuária Brasileira, v.42, p.1307-1317, 2007.

HUE, N.V. Sewage sludge. In: RECHCIGH, J.E. (Ed.). Soil amendments and environmental quality. Boca Raton: CRC Press, 1995. p.199-248.

LABORATÓRIO NACIONAL DE REFERÊNCIA VEGETAL LANARV. Análise de corretivos, fertilizantes e inoculantes: métodos oficiais. Brasília: Ministério da Agricultura, 1983. 140p.

LÓPEZ-MOSQUERA, M.E.; MOIRÓN, C.; CARRAL, E. Use of dairy-industry sludge as fertiliser for grasslands in Northwest Spain: heavy metals level in the soil and plants. Resource, Conservation and Recycling, v.30, p.95-109, 2000.

MALAVOLTA, E.; VITTI, G.C.; OLIVEIRA, S.A. Avaliação do estado nutricional de plantas: princípios e aplicações. 2.ed. Piracicaba: Potafos, 1997. 319p.
MANTOVANI, J.R.; CRUZ, M.C.P.; FERREIRA, M.E.; ALVES, W.L. Extratores para avaliação da disponibilidade de metais pesados em solos adubados com vermicomposto de lixo urbano. Pesquisa Agropecuária Brasileira, v.39, p.371-378, 2004.

MARQUES, M.O.; MELO, W.J.; MARQUES, T.A. Metais pesados e o uso de biossólidos na agricultura. In: TSUTIYA, M.T.; CAMPARINI, J.B.; ALEM SOBRINHO, P.; HESPANHOL, I.; CARVALHO, P.C.T.; MELFI, A.J.; MELO, W.J.; MARQUES, M.O. (Ed.). Biossólidos na agricultura. São Paulo: Sabesp, 2001. p.365-404.

MARTINÉZ, C.E.; McBRIDE, M.B. Solubility of $\mathrm{Cd}^{2+}, \mathrm{Cu}^{2+}$, $\mathrm{Pb}^{2+}$, and $\mathrm{Zn}^{2+}$ in aged coprecipitates with amorphous iron hydroxides. Environmental Science and Technology, v.32, p.743-748, 1998.

MATTIAZZO, M.E.; BERTON, R.S.; CRUZ, M.C.P. Disponibilidade e avaliação de metais pesados potencialmente tóxicos. In: FERREIRA, M.E. (Ed.). Micronutrientes e elementos tóxicos na agricultura. Jaboticabal: CNPq: Fapesp: Potafos, 2001. 599p.

McBRIDE, M.B. Toxic metal accumulation from agricultural use of sludge: are USEPA regulations protective? Journal of Environmental Quality, v.24, p.5-18, 1995.

MELO, V.P.; BEUTLER, A.N.; SOUZA, Z.M.; CENTURION, J.F.; MELO, W.J. Atributos físicos de Latossolos adubados durante cinco anos com biossólido. Pesquisa Agropecuária Brasileira, v.39, p.67-72, 2004.

NASCIMENTO, C.W.A.; BARROS, D.A.S.; MELO, E.E.C.; OLIVEIRA, A.B. Alterações químicas em solos e crescimento de milho e feijoeiro após aplicação de lodo de esgoto. Revista Brasileira de Ciência do Solo, v.28, p.385-392, 2004.

OLIVEIRA, F.C.; MATTIAZZO, M.E. Metais pesados em Latossolo tratado com lodo de esgoto e em plantas de cana-deaçúcar. Scientia Agricola, v.58, p.581-593, 2001.

PIRES, A.M.M.; MATTIAZZO, M.E. Biosolids conditioning and the availability of $\mathrm{Cu}$ and $\mathrm{Zn}$ for rice. Scientia Agricola, v.60, p.161-166, 2003.

QUAGgIO, J.A. Acidez e calagem em solos tropicais. Campinas: Instituto Agronômico, 2000. 111p.

RAIJ, B. van; ANDRADE, J.C.; CANTARELLA, H.; QUAGGIO, J.A. Análise química para avaliação da fertilidade de solos tropicais. Campinas: Instituto Agronômico, 2001. 285p.

RAIJ, B. van; CANTARELLA, H.; QUAGGIO, J.A.; FURLANI, A.M.C. Recomendações de adubação e calagem para o Estado de São Paulo. 2.ed. Campinas: Instituto Agronômico; Fundação IAC, 1996. 285p. (Boletim Técnico, 100).

SILVEIRA, M.L.A.; ALLEONI, L.R.F.; GUILHERME, L.R.G. Biosolids and heavy metal in soils. Scientia Agricola, v.60, p.793-806, 2003. 